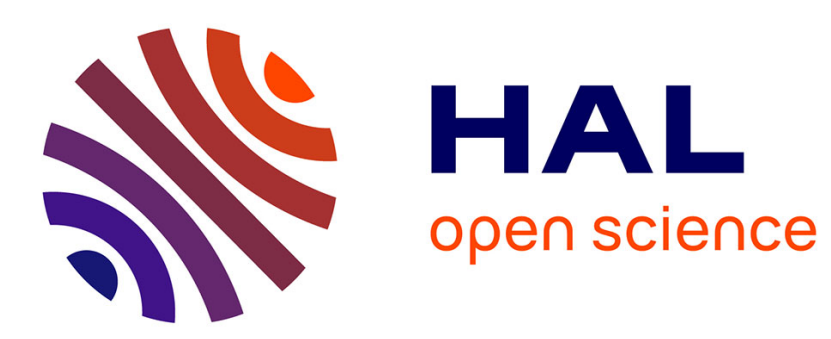

\title{
Decoding the Grid Cells for Metric Navigation Using the Residue Numeral System
}

\author{
Cécile Masson, Benoît Girard
}

\section{To cite this version:}

Cécile Masson, Benoît Girard. Decoding the Grid Cells for Metric Navigation Using the Residue Numeral System. Second International Conference on Cognitive Neurodynamics, Nov 2009, Hangzhou, China. 10.1007/978-90-481-9695-1_73 . hal-00396447

\section{HAL Id: hal-00396447 https://hal.science/hal-00396447}

Submitted on 18 Jun 2009

HAL is a multi-disciplinary open access archive for the deposit and dissemination of scientific research documents, whether they are published or not. The documents may come from teaching and research institutions in France or abroad, or from public or private research centers.
L'archive ouverte pluridisciplinaire HAL, est destinée au dépôt et à la diffusion de documents scientifiques de niveau recherche, publiés ou non, émanant des établissements d'enseignement et de recherche français ou étrangers, des laboratoires publics ou privés.

\section{(1) (1) $\$$}

Distributed under a Creative Commons Attribution - NonCommercial - NoDerivatives| 4.0 


\title{
Decoding the Grid Cells for Metric Navigation Using the Residue Numeral System
}

\author{
Cécile Masson* \\ Benoît Girard \\ ISIR, UMR 7222, UPMC - CNRS, F-75252 Paris Cedex 05, France \\ benoit.girard@isir.fr
}

June 18, 2009

\begin{abstract}
Knowing that the grid cells in the dorso-lateral band of the medial enthorinal cortex are a necessary component of rat's ability to perform path integration and homing, we explore the idea proposed by Fiete et al. (2008, J. Neurosci., 28(27):6858) that they might encode the coordinates of the rat in a Residue Number System. We show that the decoding network based on a gradient descent they propose to use for that purpose does not operate satisfactorily, and thus propose a network using a direct approach derived from the Chinese Remainder Theorem.
\end{abstract}

\section{Introduction}

Rodents are able to directly come back to their departure point after having explored an unknown environment, even in the absence of visual or other allocentric cues, exhibiting the so-called homing navigation strategy [3]. They achieve this using path integration (or deadreckoning), i.e. the integration of self-movement information derived from proprioceptive inputs, efferent copy of movement orders and vestibular information, so as to continously estimate their position relative to their departure point.

The neural substrate of this integration mechanism is thought to be the recently discovered grid cells (GC) [7] in the dorso-lateral band of the medial enthorinal cortex (dMEC). These cells fire according to a repeating equilateral triangular pattern (fig. 1, left) in the locomotion plane, characterized by a given period (the distance between two maximal response sites) and a given orientation (of one of the triangle sides with regard to an allocentric reference). Nearby cells in the dMEC have the same period and orientation but a different phase and are thus thought to belong to the same grid, while increasing grid sizes have been observed when recording cells along a dorsal to ventral axis. This spatial pattern of activity takes into account self-movement information, as it has been shown that it is preserved in absence of

${ }^{*}$ C.M. acknowledges the support of the EC Framework 6 ICEA project (Integrating Cognition, Emotion and Autonomy), IST-4-027819-IP 

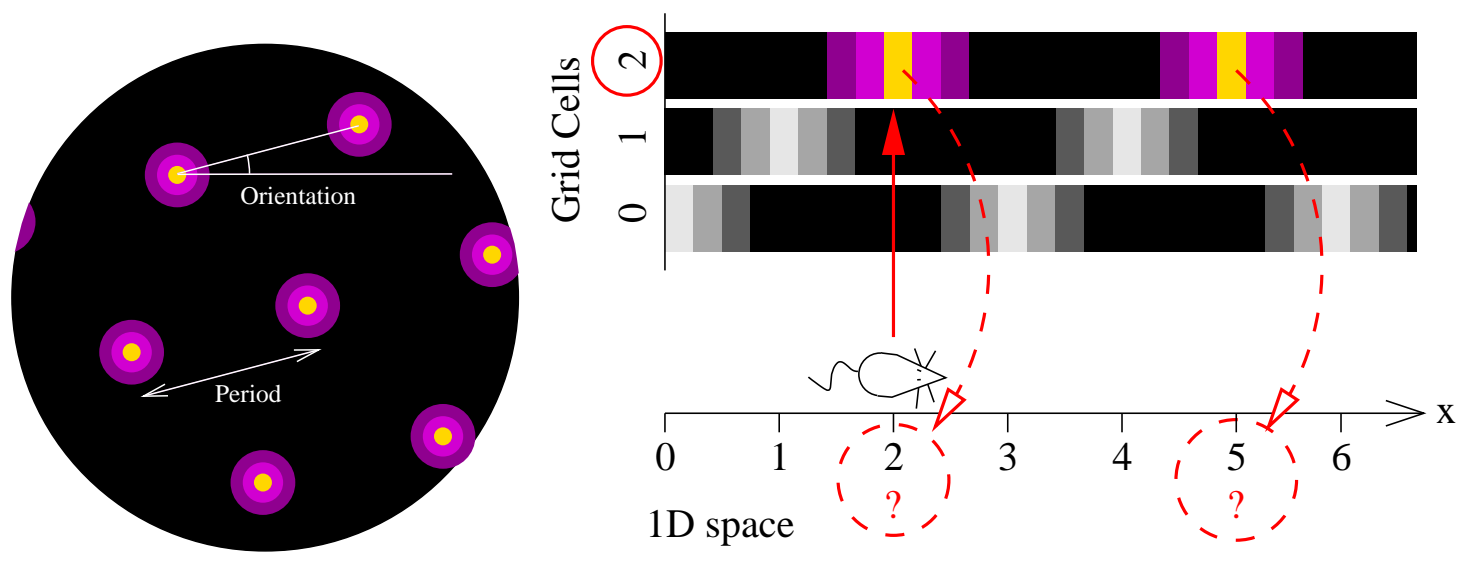

Figure 1: Left: Grid cell firing pattern schema. A grid cell recorded while the rat locomotes (here in a circular arena) fires with an equilateral triangular pattern characterized by a period and an orientation. Right: Grid cells as a modulo operator. Considering the $1 \mathrm{D}$ case, with a grid of period 3 , the current rat position is encoded (plain arrow) by neuron 2 , but neuron 2 represents all positions 2 modulo 3 (hashed arrows), adapted from [4].

any visual cue, despite a spreading of the bumps of activity, caused by accumulation of errors by the integration process.

The dMEC is an essential part of the path integration/homing system, as it has been shown that animals with an enthorinal lesion are unable to perform homing [10]. Interestingly, the hippocampus does not seem to be an essential component of path integration, as hippocampectomized rats can still perform homing [1]. Consequently, the intrinsically metric homing navigation strategy does not require functional place cells -that are a topological code of the rat position rather than a metric one- to operate. How to perform homing using the GC activity solely is a yet unanswered question, as most existing models of navigation including the GCs (like the very first one [6]) use them to generate place cells, which can then be exploited to navigate with topographical strategies, like place recognition triggered response or path planning (see $[8,9]$ for reviews on this matter). A computationel model of homing should be able to extract directly from the grid cells the current position with regard to the departure point, so as to be able to generate the opposite locomotor command.

In the following, we propose a computationel model able to extract directly from the activity of a set of grid cells the current coordinates of the animal, that can be used to control the homing behavior.

\section{Model}

\subsection{The initial idea}

In a recent paper, Fiete et al. [4] proposed a new way to interpret the GC activity: they could be considered as performing the $2 \mathrm{D}$ equivalent of modulo operator. When considering one axis of a given grid, the currently most active neuron in this grid provides the residue of the division of the current coordinate of the rat on this axis by the grid period (fig. 1, 

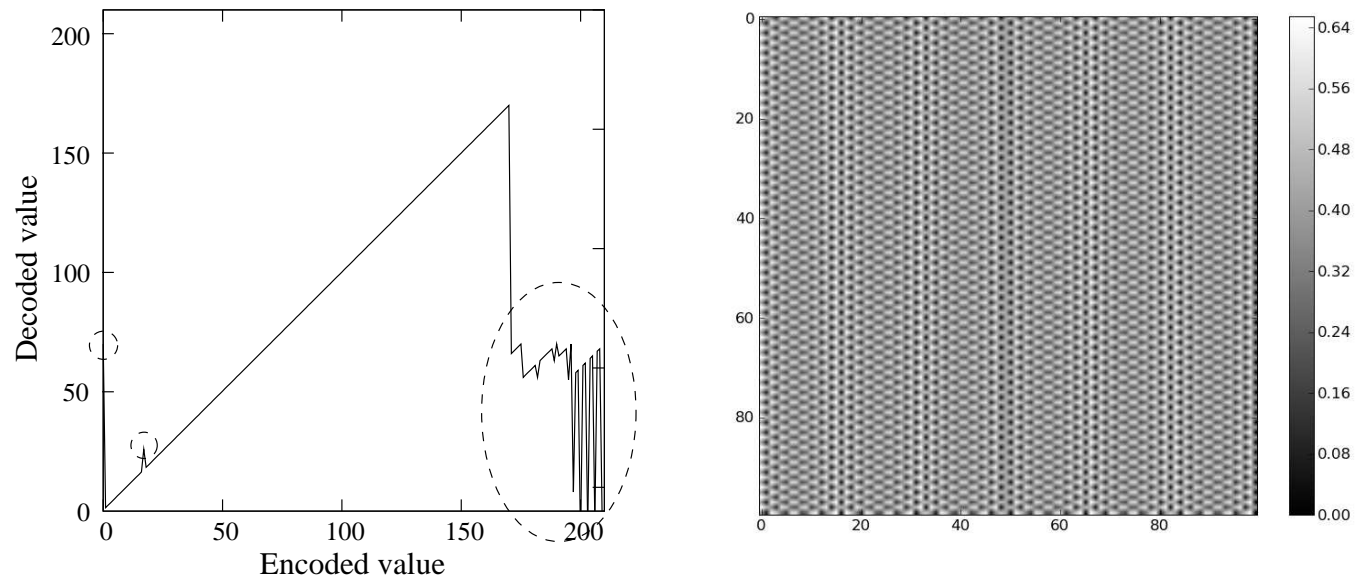

Figure 2: Left: The Sun \& Yao [13] model fails to decode correctly all the encoded values, errors, away from the diagonal, are highlighted by hashed ellipses. Right: Decoding errors (in $\mathrm{cm}$ ) generated by our model on a $100 \times 100 \mathrm{~m}$ surface.

right). The information from one grid allows to locate the animal modulo this grid's period, but the information provided by a set of $N$ grids of periods $\left(\lambda_{1}, \ldots, \lambda_{N}\right)$ corresponds to a well known number encoding system, the Residue Number System (RNS). The RNS is based on the Chinese Remainder Theorem (CRT) which states that given a set of residues $\left(r_{1}, \ldots, r_{N}\right)$ and a set of pairwise coprimes $\left(\lambda_{1}, \ldots, \lambda_{N}\right)$ (with $\Lambda=\prod_{i=1}^{N} \lambda_{i}$ ), there exist a unique integer $x$ modulo $\Lambda$ so that $\forall i \in[1, N], x \equiv r_{i} \quad\left(\bmod \lambda_{i}\right)$. Thus, given the $N$ aformentionned $\lambda_{i}$ any number in $\left[0, \Lambda\right.$ [ can be encoded as the set of residues $r_{i}$. Note that this can be generalized to non coprime pairwise periods, in which case the $\Lambda$ is the least common multiple $(\mathrm{lcm})$ of the periods. If we consider a set of grids of identical orientation and different periods, and use them as modulo operators, one should be able to extract the coordinate $x$ of the animal on one of their axes. Doing this operation on two axes of these grids provides a coordinate system sufficient to locate the animal in space, as long as it does not travel distances exceeding the periods' lcm.

Fiete et al. did not provide a computational implementation of their idea, but suggested to decode the activity of a set of grids using a neural network model proposed by Sun \& Yao [13]. However, this model computes $x$ by performing a gradient descent on an discontinuous energy function with multiple local minima:

$$
E(r)=\sum_{i=1}^{N} \frac{1}{2}\left|x \bmod \lambda_{i}-r_{i}\right|^{2}+\frac{1}{2 A R} x^{2}
$$

Such a function being unadequate for a gradient descent, it work quite poorly as it easily get stuck in local minima. For example, with a set of periods $2,3,5,7$, fig. 2 (left) shows the decoding of all the positions in $[0, \Lambda=210$ [, where numerous values are not correctly decoded.

\subsection{TRC-based model}

We propose a decoding scheme based on an explicit use of the basic mathematics associated to the CRT. Consider the $\hat{\lambda}_{i}=\frac{\Lambda}{\lambda_{i}}=\prod_{j \neq i} \lambda_{j}$ : they are pairwise coprimes, and according to 


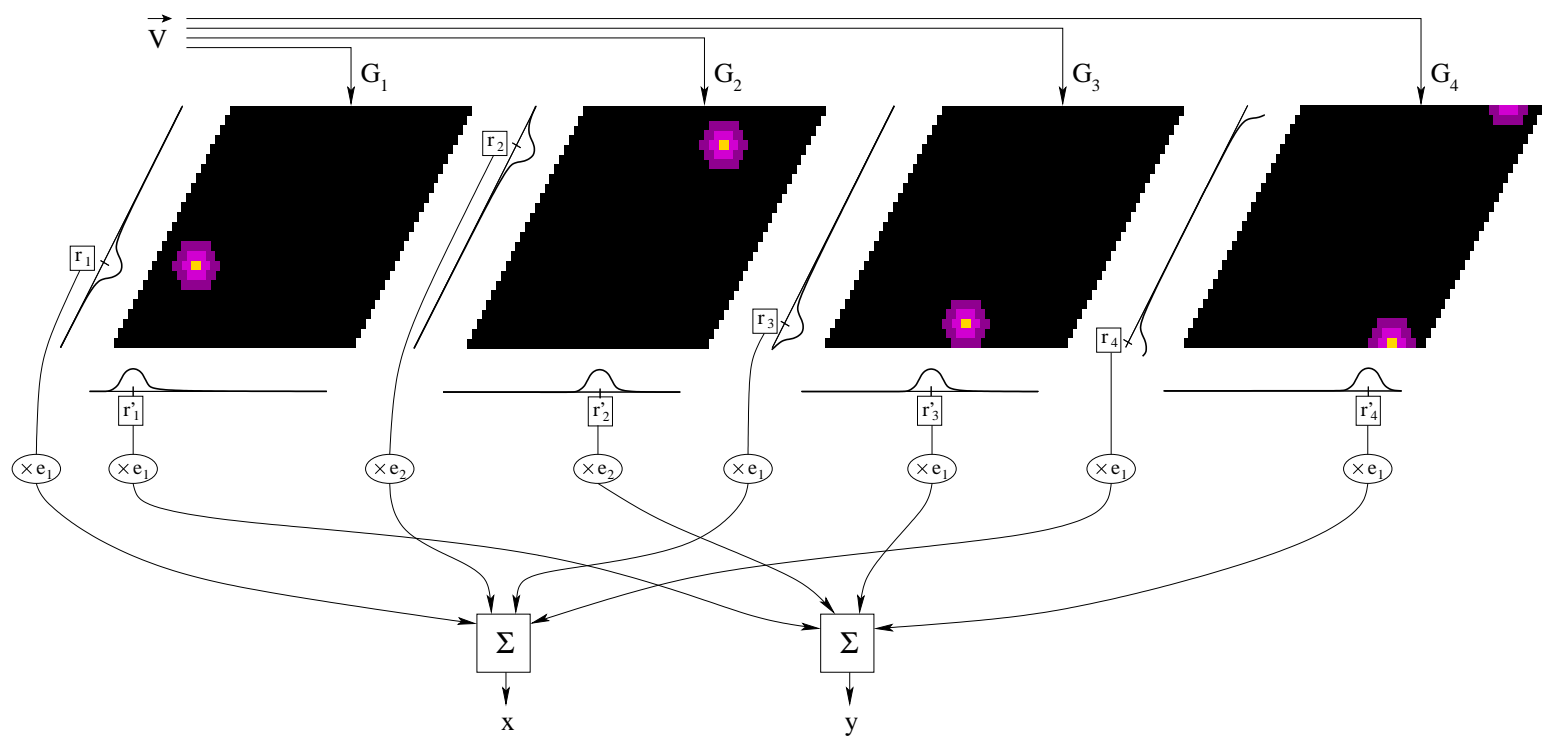

Figure 3: Grid cells decoding model. The speed $V$ of the simulated animal displaces the activity in the four grids $(25 \times 25$ neurons $)$, the gains $G_{i}$ define the grid periods $(38,50,62$ and $74 \mathrm{~cm})$. The circular barycenters $r_{i}$ and $r_{i}^{\prime}$ of the grid activities projected on one of their axes are the residues used with the $e_{i}$ weights to decode the coordinates $\left(x, x^{\prime}\right)$ (note that this reference frame is not orthogonal).

Bezout's theorem, $u_{i}$ and $v_{i}$ exist such that $u_{i} \lambda_{i}+v_{i} \lambda_{i}=1$. If we define $e_{i}=v_{i} \hat{\lambda}_{i}, x$ can be computed as the following weighted sum:

$$
x=\sum_{i=0}^{N} e_{i} r_{i}
$$

A similar solution holds when using non-pairwise coprime periods.

We built a computationel model to show the efficiency of the Fiete et al. RNS idea, using the direct RNS computation (eqn. 2). This model (fig. 3) operates in 2D and uses real residue values extracted from grid cells. It uses velocity inputs provided to 4 grids, based on the model proposed in [12]. These grids have realistic periods of $38 \mathrm{~cm}, 50 \mathrm{~cm}, 62 \mathrm{~cm}$ and $74 \mathrm{~cm}$, theoretically allowing the unique decoding of values over more than $5 \mathrm{~km}$; they all have the same orientation, which also seems to be realistic [2]. The position of the maximum activity on two of the three axes of the grids is obtained by summing the activity of all cells projected on these axes and computing the circular barycenters of the resulting vectors. These values correspond the residues $r_{i}$, which are then sent to two neurons, whose synaptic weights were set to $e_{i}$.

The simulations performed with this model show that a simulated rat is able to compute its position in 2D. The errors, caused by the discretization of the grids, have an average of $0.39 \mathrm{~cm}(s t d=0.19)$ (fig. 2 , right), a value which seems acceptable with regards to the size of a rodent. 


\section{Discussion \& Conclusion}

Based on the idea - proposed by Fiete et al. [4] - that the grid cells could be considered as an encoder of the position of animal using a residue number system, we propose a computational model performing the decoding of this position, to be used as a command for the locomotor system in a homing navigation strategy. We also establish that the residue-to-decimal decoder proposed in [13] does not operate efficiently.

Our simulations were conducted without noise in the speed measurements. In reality, such noise affects the grid updating and is fought with resets of the grid activity in places identified by learnt allocentric cues [7]. Such stabilization mechanisms are present in most GC models, and are either based on direct visual inputs or on interactions with the multimodal place cells [11]. This does not affect the validity of proposed decoding scheme, which should of course be connected to a grid cell model including a stabilization mechanism.

Finally, a computational model -anterior to the discovery of the grid cells [5]-proposed to learn the coordinates corresponding to each place cell of a hippocamp model, so as to thereafter enable metric navigation. We showed here that the grid cells, providing inputs to the place cells, are sufficient to perform metric navigation, with the advantage that no prior associative learning phase is necessary, in accordance with observations of homing behavior performed by rodents in new and unexplored environments.

\section{References}

[1] S. Alyan and B.L. McNaughton. Hippocampectomized rats are capable of homing by path integration. Behavioral Neuroscience, 113(1):19-31, 1999.

[2] C. Barry, R. Hayman, N. Burgess, and K. Jeffery. Experience-dependent rescaling of entorhinal grids. Nature Neuroscience, 10(6):682-684, 2007.

[3] A.S. Etienne and K.J. Jeffery. Path integration in mammals. Hippocampus, 14(2):180192, 2004.

[4] I.R. Fiete, Y. Burak, and T. Brookings. What grid cells convey about rat location. Journal of Neuroscience, 28(27):6858, 2008.

[5] D.J. Foster, R.G.M. Morris, and P. Dayan. A model of hippocampally dependent navigation, using the temporal difference learning rule. Hippocampus, 10(1):1-16, 2000.

[6] M.C. Fuhs and D.S. Touretzky. A spin glass model of path integration in rat medial entorhinal cortex. Journal of Neuroscience, 26(16):4266-4276, 2006.

[7] T. Hafting, M. Fyhn, S. Molden, M.B. Moser, and E.I. Moser. Microstructure of a spatial map in the entorhinal cortex. Nature, 436:801-806, 2005.

[8] B.L. McNaughton, F.P. Battaglia, O. Jensen, E.I. Moser, and M.B. Moser. Path integration and the neural basis of the'cognitive map'. Nature Reviews Neuroscience, 7(8):663-678, 2006.

[9] E.I. Moser, E. Kropff, and M.B. Moser. Place cells, grid cells, and the brain's spatial representation system. 2008. 
[10] C. Parron and E. Save. Evidence for entorhinal and parietal cortices involvement in path integration in the rat. Experimental Brain Research, 159:349-359, 2004.

[11] D. Samu, P. Erös, B. Ujfalussy, and T. Kiss. Robust path integration in the entorhinal grid cell system with hippocampal feed-back. Biological cybernetics, 2009.

[12] D. Sheynikhovich. Spatial navigation in geometric mazes : a computational model of rodent behavior. PhD thesis, EPFL, 2007.

[13] H. Sun and T. Yao. A neural-like network approach to residue-to-decimal conversion. In IEEE International Conference on Neural Networks, volume 6, pages 3883-3887, 1994. 